\title{
Based Fractional Lower-Order Statistics Detection of Envelop Modulation for Underwater Acoustic
}

\author{
ZHANG An-qing \\ Dept. of Information and Communications Engineering \\ Dalian Naval Academy \\ Dalian, China \\ e-mail: annavy@sina.com
}

\author{
WU Qing-hua \\ Dept. of Information and Communications Engineering \\ Dalian Naval Academy \\ Dalian, China \\ e-mail: annavy2@sina.com
}

\begin{abstract}
Traditional underwater target noise signal modulation spectrum analysis methods is based on the secondorder statistics characteristics in the Gaussian noise environment, but in most cases underwater acoustic signals, such as ship-radiated noise and marine environment noise, have pulse characteristic that subjecting to $\alpha$ stable distribution typically. Under this condition the second-order statistics of the modulation spectrum analysis algorithm performance not only be declined, but also can not be stable convergence any more. Aiming at this problem, the fractional lower order statistics of the target signal modulation spectrum analysis method was proposed, and technique for choosing order of fractional was debated. Simulation and experimental data results show that proposed method is robust.
\end{abstract}

Keywords- $\alpha$ stable distribution; fractional lower-order statistics; underwater Acoustics; fractional low-order spectrum of symmetrical correlation function

\section{INTRODUCTION}

Modulation noise generated by the underwater targets is major signal for passive sonar target identification. The target characteristics can be extracted by analyzing the received underwater target acoustic signals' DEMON (Detection of Envelope the Modulation on Noise) spectrum. The methods include underwater acoustic signal's higherorder spectrum analysis, wavelet transform spectrum analysis, line enhancement technology and so on ${ }^{[1-4]}$. The above methods work well in Gaussian noise environment, but the underwater environment noise obeys $\alpha$ stable distribution with pulse characteristics rather than Gaussian stable distribution according to a large number of experiments. For example, literature [5] indicated the ocean environment noise is mostly $\alpha$ stable distribution with $1.6<\alpha<1.8$. Traditional DEMON spectrum analysis performance may be decline because underwater environment noise obeys the stable distribution. A new DEMON analysis method is needed that adapts to $\alpha$ stable distribution in order to improve the robustness of the analysis system.

\section{II. $\quad \alpha$ STABLE DISTRIBUTION AND FRACTIONAL LOWER ORDER STATISTICS}

Random variables $X$, that exists parameters $0<\alpha \leq 2$, $\gamma \geq 0,-1 \leq \beta \leq 1$, and the real number $a$, are defined as $\alpha$ stable distribution ${ }^{[7]}$ by its characteristic function as follows (1).

$$
\begin{gathered}
\phi(t)=\exp \left\{j a t-\gamma|t|^{\alpha}[1+j \beta \operatorname{sgn}(t) \omega(t, \alpha)]\right\} \\
\omega(t, \alpha)=\left\{\begin{array}{cc}
\tan (\alpha \pi / 2) & \alpha \neq 1 \\
(2 / \pi) \log |t| & \alpha=1
\end{array},\right. \\
\operatorname{sgn}(t)=\left\{\begin{array}{cc}
1 & t>0 \\
0 & t=0 \\
-1 & t<0
\end{array}\right.
\end{gathered}
$$

where, $\alpha \in(0,2]$ is characteristic exponent, it measures the "thickness" of the tails of the density function and can take any value in the interval $0<\alpha \leq 2$. The small value of $\alpha$ implies considerable probability mass in the tails of the distribution. When $\alpha=2$, the relevant stable distribution is Gaussian. The $\gamma$ is called dispersion. The $\beta$ is the index of skewness. It can only take values in the interval $-1<\beta<1$. $a$ is location parameter.

Therefore, random variables $X$ with $\alpha$ stable distribution ( $a=0)$ has (3)

$$
\begin{aligned}
& E\left[|X|^{p}\right]= \\
& \begin{cases}\frac{2^{p+1} \Gamma\left(\frac{p+1}{2}\right) \Gamma(-p / \alpha)}{\alpha \sqrt{\pi} \Gamma(-p / 2)} \gamma^{p / \alpha} & \text { if } 0<p<\alpha \\
\infty & \text { if } \quad p \geq \alpha\end{cases}
\end{aligned}
$$

Where $\Gamma(\bullet)$ is the gamma function.

Obviously, it is known that a real non-Gaussian $\alpha$ stable distribution random variable with zero location parameter has finite fractional lower order moment. By reason of the second order moment of a $S \alpha S$ process with $0<\alpha<2$ does not exist, the performance of underwater acoustic for detection and analysis which is designed based on second order statistical and assumption of Gaussian must be degenerate under the $\alpha$-stable distribution noise. Hence a new method with using FLOM should be adopted ${ }^{[6-7]}$.

\section{ANALYSIS OF THE DEMON SPECTRUM BASED ON FRACTIONAL LOWER ORDER STATISTICS}

Symmetric correlation function and symmetric spectrum are defined as (4) and (5): 


$$
\begin{gathered}
W_{x}(t)=\int_{-\infty}^{\infty} x(t+\tau / 2) x(t-\tau / 2) d \tau \\
F_{w x}(\omega)=\int_{-\infty}^{\infty} W_{x}(t) e^{-j \omega t} d t
\end{gathered}
$$

In the $\alpha$ stable distribution noise environment, statistical moment is the unstable convergent if the order is higher than characteristic index $\alpha$, so traditional power spectrum and symmetric spectrum is no longer suitable. Therefore, we propose new analysis methods based on fractional lower order symmetric correlation function and fractional loworder spectrum of symmetrical correlation function, which are defined as (6) and (7):

$$
\begin{gathered}
W_{x}{ }^{d}(t)=\int_{-\infty}^{\infty}[x(t+\tau / 2)]^{<B_{1}>} \cdot[x(t-\tau / 2)]^{<B_{2}>} d \tau \\
F_{w x}{ }^{d}(\omega)=\int_{-\infty}^{\infty} W_{x}{ }^{d}(t) e^{-j \omega t} d t
\end{gathered}
$$

Where $x(t)^{<B>}=\left|x(t)^{B}\right| \cdot \operatorname{sign}[x(t)], 0<B_{1}+B_{2}<\alpha \leq 2$.

According to (3), it is stable fractional lower order symmetric correlation function and fractional low-order spectrum of symmetrical correlation function that can be achieved to analyze signal spectrum compatibly when the parameters satisfy $0<B_{1}+B_{2}<\alpha \leq 2$.

A large number result of simulation analysis denotation, the select of parameters $B_{1}$ and $B_{2}$ are determined by foreand-aft signal's pulse intensity. The parameters $B_{2}$ should be reduced If above signals' impact is stronger than the later, otherwise, the parameters $B_{1}$ should be reduced, and the parameters $B_{1}$ and $B_{2}$ should meet $0<B_{1}+B_{2}<\alpha \leq 2$ as a whole.

Fractional low-order spectrum of symmetrical correlation function not only inherits higher resolution characteristics of the symmetry power spectrum but also performs very well in suppressing impulse noise. Therefore, underwater targets DEMON spectrum detection methods based on fractional low-order spectrum of symmetrical correlation function is proposed to improve on the traditional method. The algorithm steps are as follows:

First step, parameters $\alpha$ is estimated by using the methods proposed in reference [8] for underwater acoustic.

The second step, demodulation is performed via the band-pass filter and the absolute value low-pass filter.

The third step, employing N-point signal to determine parameters $B_{1}$ and $B_{2}$ of the fractional lower order symmetric power spectrum.

The fourth step, the fractional low-order spectrum of symmetrical correlation function can be extracted according to (6) and (7).

\section{SimUlATION AND ANALYSIS}

Assuming $x(t)=\sum_{i=1}^{n}\left[1+m \cdot \sin \left(2 \pi \Omega_{i} t+\varphi_{i}\right)\right] \cos \omega t+n(t)$, harmonic number $n=4$, degree of modulation $m=0.5$, harmonic frequency $\Omega_{i}=10 \cdot i, \quad \varphi_{i}$ is early phase of harmonic modulation component multiple frequency, carrier frequency $\omega=1000 \mathrm{~Hz}, n(t)$ is the $\alpha$ stable distribution environment noise, and mixed signal to noise ratio is calculated via equation $M S N R=10 \log _{10}\left(\sigma_{s}^{2} / \gamma_{n}\right)$

Experiment 1: On different mixed signal to noise ratio, traditional methods' performance comparison with the fractional low-order spectrum of symmetrical correlation function methods on the assumption that parameters $\alpha$ is the same. Fig. 1 shows the effect of underwater target's DEMON for multi-cases. Table 1 shows that the minimum MSNR (dB) statistics witch basic frequency recovery correct rates is higher than $80 \%$ are obtained by the traditional DEMON spectrum and the new method proposed, in presences of the stable distribution noise with different parameters $\alpha$ for 300 independent simulation processing.

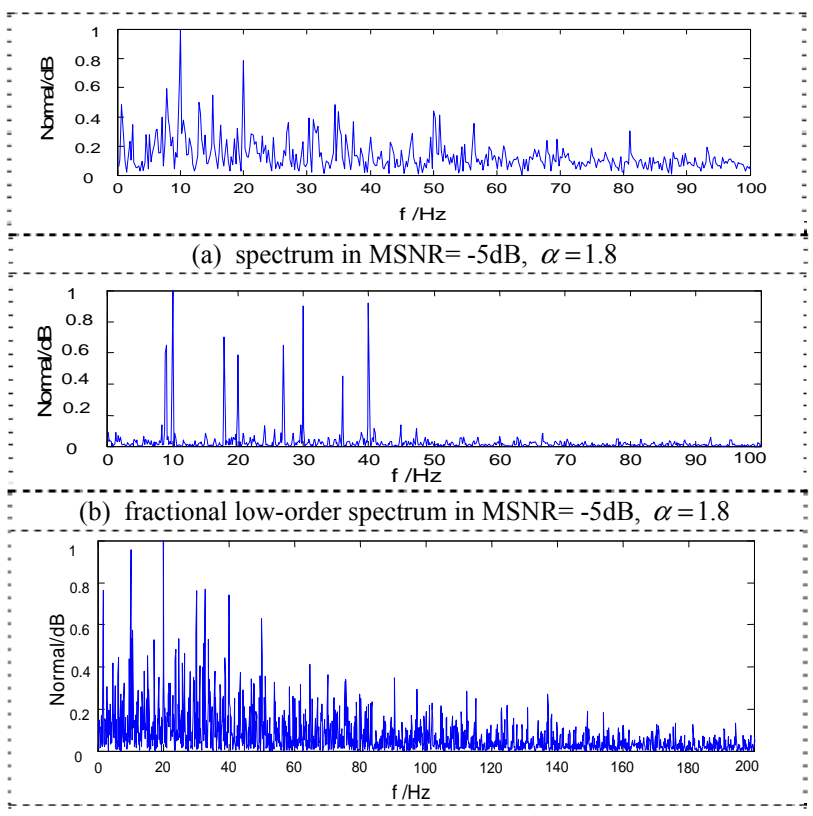

(c) spectrum in MSNR $=-9 \mathrm{~dB}, \alpha=1.6, \quad B=B_{2}=\alpha / 2$

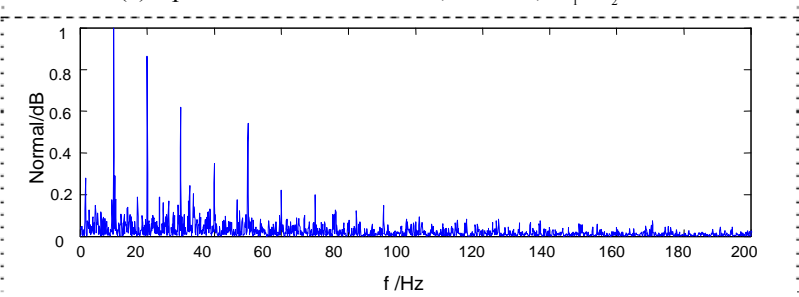

(d) fractional low-order spectrum in MSNR=$9 \mathrm{~dB}, \alpha=1.6, B_{1}=B_{2}=\alpha / 2$

Figure 1. Effect of underwater target's DEMON for multi-cases.

Seen from the TABLE I, the performances are equivalent both of the methods proposed and traditional methods for signal analysis capability in presences of Gaussian noise ( $\alpha=2)$. But in presences of $\alpha$ stable distribution noise and $\alpha<2$, it is difficult to obtain steadily modulation spectrum information for the traditional methods, whose performance decreased significantly, while the methods proposed have good performances and robustness ability to suppress impulse noise. 
TABLE I. MSNR (DB) OF BASIC FREQUENCY RECOVERY CORRECT RATES HIGHER THAN $80 \%$

\begin{tabular}{|c|c|c|c|c|c|c|}
\hline $\boldsymbol{\alpha}$ & $\mathbf{1 . 5}$ & $\mathbf{1 . 6}$ & $\mathbf{1 . 7}$ & $\mathbf{1 . 8}$ & $\mathbf{1 . 9}$ & $\mathbf{2 . 0}$ \\
\hline $\begin{array}{l}\text { DEMON } \\
\text { spectrum }\end{array}$ & 6 & 5 & -3 & -8 & -12 & -15 \\
\hline $\begin{array}{l}\text { fractional } \\
\text { low-order } \\
\text { spectrum }\end{array}$ & -9 & -10 & -13 & -13 & -15 & -15 \\
\hline
\end{tabular}

Experiments 2: Assuming $\alpha$ stable distribution noise with $\alpha=1.6$ and the presences $-6 \mathrm{db}$ for signal to noise ratio, the performance is analyzed for difference parameters B. The results are shown as Fig. 2.

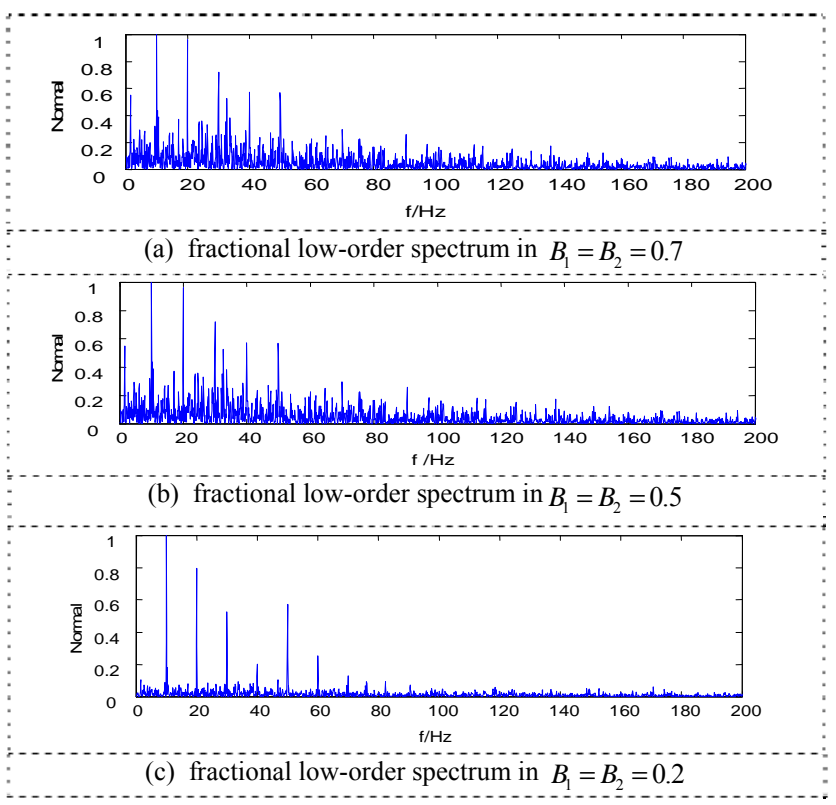

Figure 2. fractional low-order spectrum in difference parameters B

The Fig. 2 demonstrates fractional B lower the better for restrain frequency of noise, but it causes some harmonic loss. Simulations indicate the spectrum of estimating is good results for selecting $0.4<B_{1}+B_{2}<(\alpha-0.4)$.

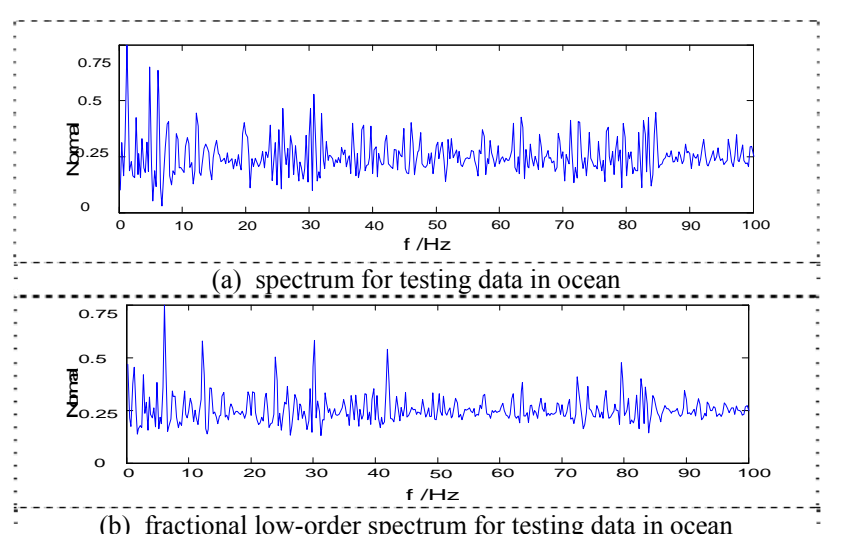

(b) fractional low-order spectrum for testing data in ocean

Figure 3. Effect comparing between (a) traditional and (b) proposed method.
Experiments 3: Ocean experimental data analysis. A target radiated noise data samples, the sampling frequency $25 \mathrm{KHz}$, the length of time sampling $4 \mathrm{~s}$.Ocean experimental data obeys to $\alpha$ stable distribution with $1.65<\alpha<1.75$, the DEMON spectrums are shown in Fig.2:

In Fig. 3 (a), it is difficult to achieve the frequency information of the underwater target signal with the traditional analysis methods while more clearly target line spectrum information could been achieve with the methods proposed (The figure horizontal axis unit is the normalized frequency).

\section{CONCLUSIONS}

Aiming at the depressed performance of traditional DEMON spectrum analysis in $\alpha$ stable distribution noise environment, fractional low-order DEMON spectrum analysis methods is proposed to adjust to the $\alpha$ stable distribution noise environment. By analyzing simulation and ocean experimental data results, the methods proposed have the same effect of spectrum analysis compared with the traditional methods in the Gaussian noise environment. But in $\alpha$ stable distribution noise environment with the pulse features, the performance of traditional DEMON spectrum analysis methods is unstable and declines obviously while fractional low-order DEMON spectrum analysis methods with robust perform. Proposed method is worth application and generalizing.

\section{REFERENCES}

[1] BAO Zhong-hua, GONG Shen-guang, WU Zheng-guo, LI Jun. “ Line spectrum extraction of harmonic signals by using fourth-order cumulant slice spectrum", Journal of Navy University of Engineering, vol. 22(3), pp. 31-34, 2010.

[2] LI Yao-bo, REN Zhi-liang, CHEN Hong-bo. "A Method for Modulation Feature Extraction of Underwater Target Radiated Noise", Torpedo Technology, vol. 14(3), pp. 21-24, 2006.

[3] YANG Ri-jie, YANG Chun-ying, WANG Ri-hong. "Characteristic Extraction Method for Underwater Target Radiated Noise Based on Wavelet Transform ", Journal of Data Acquisition \& Processing, vol. 17(3), pp. 279-282, 2002.

[4] YANG Xi-lin, ZHOU Jin, WANG Bing-he, ZHENG Si-yi. "Application of adaptive line enhancement on testing the shipradiated noise ", Ship Science and Technology, vol. 31(3), pp. 93-95, 2009.

[5] ZHANG An-qing. "Analysis of Impulsive Noise Characteristic for Shallow Sea Channel ", Technical Acoustic, vol. 26(5), pp. 988-989, 2007.

[6] QIU Tian-shuang, LI Xiao-bing, SUN Yong-mei, WANG Hong-yu. "Fractional Lower Order $\alpha$-Stable Distribution and Issues in Its Applications", Journal of Detection \& Control, vol. 26(2), pp. 5-9, 2004.

[7] C.L.Nikias, M.Shao, "Signal Processing with Alpha-Stable Distributions and Applications", New York: John Wiley \& Sons Inc., 1995.

[8] SUN Yong-mei. "Study on the Theory and Application of Parameter Estimation and Spectral Analysis of alpha stable Distribution", Dalian, Dalian University of Technology, 2006. 